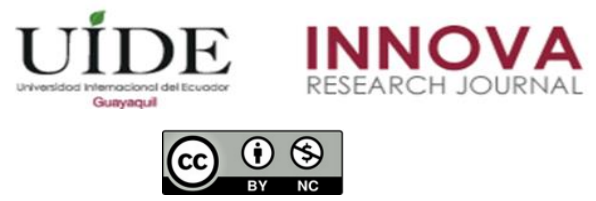

INNOVA Research Journal, ISSN 2477-9024

(Septiembre-Diciembre 2019). Vol. 4, No.3.1 pp. 42-54

DOI: https://doi.org/10.33890/innova.v4.n3.1.2019.1076

URL: http://revistas.uide.edu.ec/index.php/innova/index

Correo: innova@uide.edu.ec

\title{
Emprendimiento dinámico, crecimiento económico y política pública en el Ecuador
}

\section{Dynamic entrepreneurship, economic growth and public policy in Ecuador}

John Alexander Campuzano Vásquez

ORCID: 0000-0002-3901-3197

Samuel Efraín Chuquirima Espinoza

Wilton Eduardo Romero Black

Universidad Técnica de Machala, Ecuador

Luis Fernando Cedillo Chalaco

Consultor independiente, Ecuador

Autor para correspondencia: jcampuzano@utmachala.edu.ec; luiscedillo_analysis@hotmail.com; schuquirima@utmachala.edu.ec; weromero@utmachala.edu.ec

Fecha de recepción: 12 de julio de 2019 - Fecha de aceptación: 29 de octubre de 2019

\section{Resumen}

Desde Cantillon 1732 hasta las contribuciones actuales realizadas por distintos economistas para entender el comportamiento e implicaciones de emprender, permiten establecer el objetivo de este trabajo investigativo, el cual explora la compleja relación existente entre el emprendimiento y el crecimiento económico a partir del impulso que proporcionan las políticas públicas y programas implementados en el Ecuador desde el período 2007 - 2017. Para esta investigación se ha tomado como referencia el reporte del Doing Business 2018, el Global Entrepreneurship Monitor 2017 y las normas creadas en los últimos diez años en Ecuador que apoyan el emprendimiento. Usando el enfoque metodológico mixto con alcance descriptivo-exploratorio transversal y longitudinal, se analizaron datos presentados por el Doing Business 2018 del Banco Mundial y el Global Entrepreneurship Monitor 2017; sumándose a la revisión literaria de diferentes artículos científicos y de políticas establecidas por el gobierno ecuatoriano del período mencionado. Los principales resultados encontrados en torno a Ecuador evidencian la existencia de una relación positiva entre la actividad emprendedora y el crecimiento económico, a tal punto, que las políticas públicas tomadas desde el 2010 han generado un entorno favorable para mejorar el posicionamiento de este país en la forma de hacer negocios rentables.

Palabras claves: crecimiento económico; emprendimiento; políticas públicas; doing business; negocios rentables

\begin{abstract}
From Cantillon 1732 to the current contributions made by different economists to understand the behavior and implications of undertaking, they allow us to establish the objective of the current research, which tries to explore the complex relationship between entrepreneurship and economic growth based on the momentum that they provide the public policies and programs implemented
\end{abstract}


in Ecuador from the 2007 - 2017 period. For this research the report of the Doing Business 2018, the Global Entrepreneurship Monitor 2017 and the norms created in the last ten years in Ecuador that support have been taken as a reference entrepreneurship Using the mixed methodological approach with transversal and longitudinal descriptive-exploratory scope, data presented by the Doing Business 2018 of the World Bank and the Global Entrepreneurship Monitor 2017 were analyzed; adding to the literary review of different scientific and policy articles established by the Ecuadorian government of the mentioned period. The main results found around Ecuador show the existence of a positive relationship between entrepreneurial activity and economic growth, to such an extent that public policies taken since 2010 have generated a favorable environment to improve the positioning of this country in the way of doing profitable business.in the way of doing profitable business.

Key words: economic growth; entrepreneurship; public politics; doing business; profitable business

\section{Introducción}

En 1732, el economista irlandés Richard Cantillon describió el emprendimiento como la voluntad de los individuos de desarrollar formas de intermediación que implican el riesgo económico de una nueva empresa. Desde entonces, distintos economistas como John Stuart Mill, Jean Baptist Say, Joseph Schumpeter, y William Baumol han sido algunos de los autores que más han contribuido a nuestro entendimiento del comportamiento emprendedor y a sus implicaciones para el crecimiento económico (Minniti, 2012).

Por su parte, para Menger (1871) "el desencuentro de autores de la Escuela Austriaca, con los clásicos y los neoclásicos al negar el equilibrio de la economía y la posibilidad del completo conocimiento de toda la información por los agentes económicos; en consecuencia, existirían demandas insatisfechas, y serían pues los empresarios emprendedores, capaces de encontrar y manejar la información adecuada, los que desarrollarían bienes y servicios para satisfacer estas necesidades" (citado en Carrillo, Priede, y López, 2014, p. 56).

De hecho, este entendimiento del comportamiento emprendedor y del manejo de la información adecuada, han hecho que, en estos últimos diez años en Ecuador, se desarrollen muchas acciones de política pública coordinadas con gobiernos seccionales (municipios y prefecturas) (algunas denominadas como emblemáticas), y organizaciones no gubernamentales (ONG's) para atender la figura del emprendedor como elemento de cambio y apoyo al crecimiento económico.

Por lo que dualidad de emprendimiento y crecimiento económico, está ahora en las agendas de los gobiernos de todo el mundo, lo que permite abrir nuevas líneas de intervención centradas en el apoyo a microempresas y organizaciones de interés social o comunitario, lo que en Ecuador se viene conociendo como empresas de economía popular y solidaria (EEPS). Esta nueva orientación pública trata de fortalecer la interacción de organizaciones productivas consolidadas con las que no lo están y con las que son parte de la economía popular y solidaria en una cadena de abastecimiento de innovación y de creatividad competitiva. Por lo que el emprendimiento se convierte en un componente vital para el crecimiento de la economía en su 
conjunto, el que pasaría de depender de empresas grandes a empresas de menor tamaño, una realidad creciente en las estadísticas societarias de América Latina.

Sin embargo, no se pueden ignorar las críticas ante la falta de evidencia sobre si es necesario apostar muchos incentivos económicos gubernamentales a la relación emprendimiento y crecimiento a partir de nuevas empresas antes que las empresas predominantemente grandes (Audretsch y Fritsch, 2002; Fritsch y Mueller, 2004). En Ecuador desde la década pasada se viene discutiendo un modelo de desarrollo económico basado en empresas de rápido crecimiento, las llamadas «high-growth-firms», que en estudios de Hölzl (2010) "llama a distinguir entre la política de pequeñas y medianas empresas (PYME), que se buscan apoyar, y la política de emprendimiento, que busca apoyar solo a empresas con ambiciones de crecimiento" (citado en Coad, Daunfeldt, Hölzl, Johansson, y Nightingale, 2014, p. 93).

En este sentido se busca mediante este trabajo trata de examinar la complicada relación que existe entre el emprendimiento y el crecimiento económico a partir del fomento de políticas públicas realizadas en Ecuador desde el periodo 2007 a 2017 al ser el corte temporal más largo con el que ha contado el país bajo un solo gobierno de corte socialista, a su vez, se busca relacionar si estas políticas públicas han servido de manera eficiente al objetivo de más empleo y crecimiento; para ello se toma como referencia el reporte del Doing Business 2018, el Global Entrepreneurship Monitor (GEM 2017) y las normas creadas en los últimos diez años en Ecuador. Esta investigación tiene un enfoque descriptivo (Hernández, Fernández, y Baptista, 2014; Jaimes, Márquez, y Pernía, 2015) al considerar la información que aportan los anteriormente mencionados reportes, los que aportan datos que corresponden a un diseño transversal (Rojas, 2015).

\section{Emprendimiento}

Según Morales (2009) el emprendimiento es la mezcla de la capacidad y actitud de las personas para poner en práctica nuevos proyectos de diferente índole, haciendo énfasis en aquellos que sobresale la creatividad; es decir, la capacidad de convertir algo abstracto en físico. Por otra parte, Sánchez, García, y Mendoza (2015) mencionan que el emprendimiento es un instrumento que recoge conocimientos para conectarlos con la innovación y el crecimiento económico de un país, los cuales generan mayores riquezas para los instauradores de los negocios (Fuentelsaz y González, 2015).

Dicho fenómeno afecta cada año en todo el mundo, a aproximadamente 500 millones de individuos (Palmero, y otros, 2014), así mismo Oyarvide, Nazareno, Roldán, y Ferrales (2016) manifiestan que contribuye de forma directa al producto interno bruto, mercado interno y a las cadenas productivas que dinamizan la economía de un país, para alcanzar el progreso de los pueblos. Sin dejar de mencionar, que para conseguirlo se debe tener en cuenta la actividad emprendedora que deriva de las habilidades, actitudes y conocimientos de los individuos emprendedores (Silveira, Cabeza, y Fernández, 2016; Campuzano, Espinoza, y Cedillo, 2017).

Sin embargo, a pesar de la gran cantidad de personas que están inmiscuidas en el emprendimiento, muchas de las ocasiones estas no cuentan con los instrumentos necesarios para hacer de dichos emprendimientos ideas de negocios sustentables y sostenidos en el tiempo, entre 
las que resaltan según Fuentelsaz y González (2015): el papel de las instituciones y la calidad de dicho emprendimiento. Los que al ser insuficientes pueden llevar al fracaso y al cierre a corto plazo, dejando como secuelas cuantiosas pérdidas.

En vista de lo anterior, para no dar paso al fracaso de los emprendimientos, se habla de emprendimiento dinámico como una forma de superar las crecientes muertes en empresas con pocas capacidades de escalabilidad. El término emprendimiento dinámico que no posee de manera universal una definición como tal, aun así, Fischer y Reuber (2003) lo definen como una empresa que genera réditos superiores al $20 \%$ anual durante cinco años consecutivos. $\mathrm{O}$ en todo caso, generar beneficios superiores a sus competidores (Cantón, García, y Iuit, 2014).

Este tipo de empresa contará por los menos con quince trabajadores y un máximo de trescientos (Kantis, Angelelli, y Moori, 2004). Solo un pequeño grupo de la población empresarial cumple con dicho perfil (Kantis y Díaz, 2008). Además, este tipo de empresas, posee características de horizonte exportador, debido a que cuentan desde sus inicios con un modelo de negocio diferenciador, que ofrecen al mercado productos y servicios innovadores, desde la perspectiva de diseño y proceso tecnológico aplicado (Messina y Hochsztain, 2015).

Las empresas dinámicas, o también denominadas empresas de alto crecimiento «highgrowth-firms», en paralelo a lo dicho por Kantis y Díaz, representan tan solo una pequeña parte del total de la actividad empresarial, y por lo general son creadas en territorios de América del Norte, países de la Unión Europea, Asia (especialmente China y Japón) y Oceanía (Arteaga y Lasio, 2009), y surgen en respuesta a problemas concretos de la sociedad (Chong, 2016).

Uno de los principales factores para la existencia de empresas dinámicas, es que existan emprendedores con ideas de negocios orientadas al crecimiento, es decir, aquellos individuos que posean a más de la intención de emprender, capacidades, vocación y el potencial para hacer de sus emprendimientos empresas que crezcan significativamente con el paso del tiempo (Kantis, Gonzalo, y Álvarez, 2014), y no fracasen como los emprendimientos convencionales que se dan día a día, algo que también se asocia a la formación universitaria que poseen los emprendedores.

Dentro del contexto Latinoamericano, para hablar de emprendimiento dinámico, se debe hacer referencia en primera instancia a las pequeñas y medianas empresa (pymes), las cuales se caracterizan especialmente por poseer un número considerado de trabajadores e ingresos medianamente moderados, este tipo de organizaciones se han convertido en un órgano fundamental del sector empresarial dentro de la región de América Latina, aportando aproximadamente al crecimiento económico en un 30\% (Dini, Rovira, y Stumpo, 2014).

Este tipo de emprendimientos dinámicos, actualmente se han convertido dentro del continente en un tema de alto interés para las distintas esferas de la política pública, ya que la gesta y crecimiento de estas empresas es una fuente relevante de riqueza y oportunidades de crecimiento y desarrollo para dichos territorios, y por tanto, un factor clave para la reducción de la brecha de la pobreza (Hidalgo, Kamiya, y Reyes, 2014).

En América Latina, múltiples estudios, hacen referencia a emprendimiento dinámico como el conjunto de empresas que tienen pocos años en el mercado, y que cuyo crecimiento les 
ha permitido (gracias a la aplicación de estrategias más competitivas) pasar en el corto plazo de empresas micro a empresas pymes, permitiéndoles tener un crecimiento favorable (Kantis, Gonzalo, Federico, y Ibarra, 2014).

A priori en la etapa de gestación e iniciación de emprendimientos dentro de la región, según Kantis et al. (2014) es necesario que dichos individuos que deseen emprender cuenten con motivaciones y vocaciones que les permita hacerlo y llevar sus negocios hacia el crecimiento constante; dichos eventos, se ven directamente influenciado por diversos factores, entre los que destacan: contexto cultural, conducta emprendedora, innovación, educación, capital social, financiamiento, estructuras productivas, capital humano y políticas y regulaciones. Estas últimas, creadas en función de la orientación que quieran imprimir los gobiernos de turno, y que responden a la orientación que imprime cada gobierno con lo que se puede hablar de políticas cortoplacistas antes que decisiones de Estado.

No obstante, cabe mencionar que el emprendimiento dinámico dentro de los diferentes países de Latinoamérica, no cumple con las características necesarias y suficientes para ser considerado como tal, y esto se debe a que dichas empresas simplemente buscan mejorar su capital y producción en términos no disruptivos e innovadores, viéndose empalidecidos por países avanzados en materia empresarial (Kantis, Federico, y Ibarra, 2014).

Para Montoya (2015) en paralelo al inciso anterior, indica que la región del sur, pese a los esfuerzos que realiza y ha venido realizando desde hace algo más de una década en cuanto a mejorar las redes empresariales, el contacto con el consumidor, la mejora de los procesos productivos, la tecnología y el conocimiento a través de la especialización; los resultados son pocos sólidos en contraste a los países desarrollados, quedándose muchas veces en pequeñas empresas y no transformarse en empresas de categoría dinámica.

Dentro del Ecuador las empresas consideradas como dinámicas o pymes, representan única y aproximadamente el 9,3\% del global del sector empresarial, valor que resulta poco atractivo, el que además presenta bajas participaciones en exportaciones, industrialización y generación de mejores condiciones de empleo para los ecuatorianos (Padilla, Lascano, y Ramiro, 2018).

En la misma línea, Garcés (2015) menciona que dichas debilidades del sector empresarial dinámico ecuatoriano se lo debe a la generación de políticas públicas superficiales, que hacen nulo el esfuerzo de los emprendedores al momento de querer acrecentar sus negocios, manteniendo relegados la competitividad, desarrollo empresarial y productividad.

Por lo que lograr un impacto económico positivo en este tipo de empresas según varios autores requiere de una política pública que promueva y fortalezca el emprendimiento, considerando las imperfecciones o fallas del mercado que en ocasiones pone barreras para la entrada de nuevas iniciativas. Hay que considerar que el mercado ecuatoriano está dominado por pocos grupos económicos que se pueden constituir en obstáculos, lo que exige algún tipo de regulación, en el caso del Ecuador en el 2011 se cuenta con la Ley de Control de Poder de Mercado (antimonopolio). 
Esta ley establece en su artículo 1, referido al objeto: el evitar, prevenir, corregir, eliminar y sancionar el abuso de operadores económicos con poder de mercado; la prevención, prohibición y sanción de acuerdos colusorios y otras prácticas restrictivas; el control y regulación de las operaciones de concentración económica; y la prevención, prohibición y sanción de las prácticas desleales, buscando la eficiencia en los mercados, el comercio justo y el bienestar general y de los consumidores y usuarios, para el establecimiento de un sistema económico social, solidario y sostenible (Asamblea Nacional del Ecuador, 2012). Esta ley está se enmarca en la Constitución de la República del Ecuador del 2008, Arts. 283, 304, 335, 336 y guarda relación con el código orgánico de la producción, comercio e inversiones, Arts. 5.

Con el marco legal se han creado instrumentos de apoyo, como la Agenda de la Transformación Productiva la que contiene políticas transversales y sectoriales que buscan hacer efectivas estrategias de innovación y emprendimiento planteadas por el gobierno nacional de cambio de una matriz productiva sin mayor valor agregado a otra orientada a la exportación.

Las políticas públicas reconocen que la falta de apoyo financiero es una de las causas de que muchos emprendimientos no se lleguen a ejecutar, y para ello, se plantea que corresponde al Estado potenciar el financiamiento de las micros y pequeñas empresas en todo el territorio nacional, según se establece el artículo 67 del Código de la Producción.

Las políticas planteadas y descritas se instrumentan a través de varios programas que orientan y clasifican el destino de los recursos que el Estado ecuatoriano decide invertir, de forma soberana, para el desarrollo de sectores estratégicos y, en muchos de los casos, en las denominadas Zonas Estratégicas de Desarrollo (ZEDES). Forma parte de sus políticas el incremento de los recursos que financien proyectos con capitales de riesgo, así como también, el establecimiento de capital semilla.

Entre los programas propuestos por el Estado ecuatoriano durante los años 2010 y 2011 para fomentar el emprendimiento se tienen los siguientes: ProduceEcuador, mediante el cual se intenta diagnosticar potencialidades y oportunidades regionales y provinciales, y son los mismos sectores los que dan prioridad a sus principales actividades. Como complemento, el programa CreeEcuador, aportará capital de riesgo con montos que oscilan entre $\$ 500.000$ y \$2.000.000, así mismo se cuenta con EmprendeEcuador, que es un programa de fomento dirigido al empresarioemprendedor, el que brinda información sobre emprendimiento, asesorías, validación de proyectos y capital para la puesta en marcha de micro emprendimientos. 


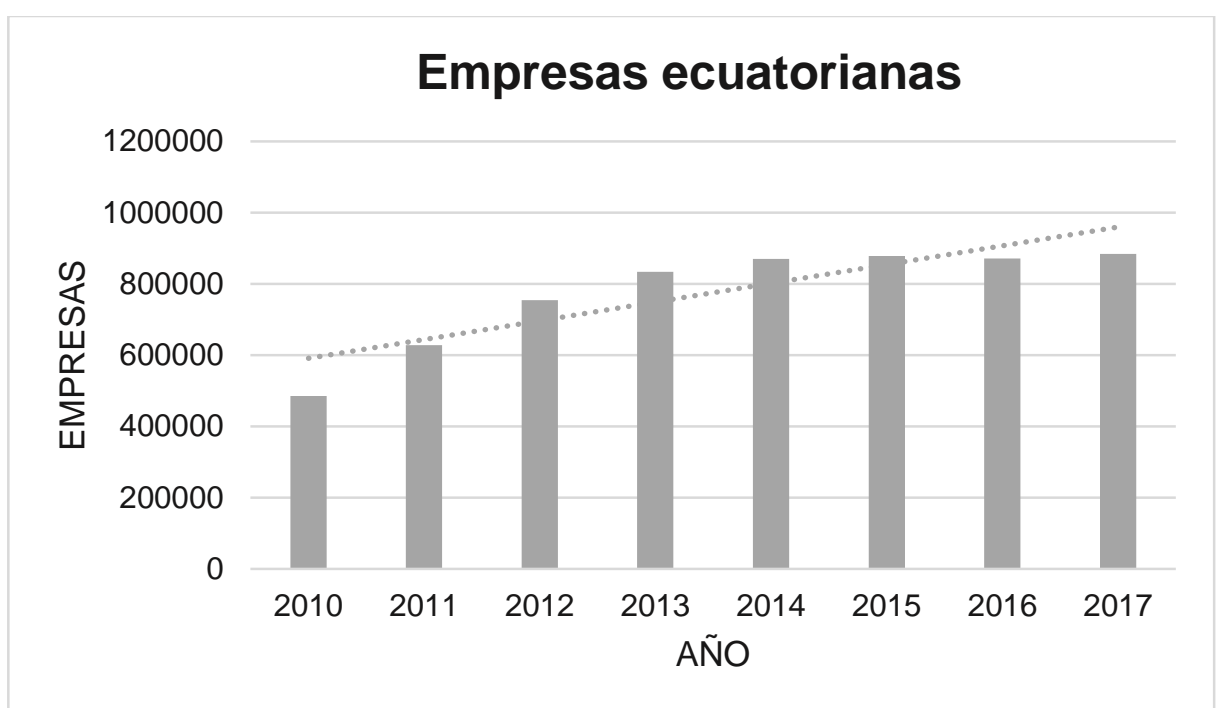

Ilustración \#1 Desde el fomento de políticas públicas (año 2010) para el crecimiento del emprendimiento, Ecuador se ha visto favorecido año a año con el nacimiento de nuevas empresas en los distintos sectores estratégicos (agrícola, comercio, inmobiliaria, manufactura y construcción), presentando ceteris paribus una tendencia positiva para los años subsecuentes. Tomado de (INEC, 2017), Elaborado por los autores.

InnovaEcuador, cierra el círculo de los programas impulsados por el gobierno del periodo 2007 al 2017 periodo sin lugar a dudas de mucho aporte al emprendimiento, siendo un programa que promueve la innovación en la empresa, la diversificación productiva, y la eficiencia logística mediante el cofinanciamiento de proyectos innovadores generadores de nuevos productos o externalidades.

La presencia de estos programas y de un marco regulatorio favorable para minimizar las fallas del mercado y para aumentar la presencia de emprendedores, debe tener resultados visibles luego de cierto tiempo, la tabla 1 recoge los reportes que entrega anualmente el Doing Business que son un reflejo del accionar del sector gobierno y sus regulaciones en la facilidad de hacer negocios. Se puede observar tomando desde el año 2013 al 2018 considerando los rezagos que siempre presentan las decisiones de política pública en los resultados, que hay significativos avances en el ranking, aunque a lo largo de esos años de observación el Ecuador solo supera a Bolivia y Venezuela, quedando muy por debajo de sus competidores más cercanos como Perú y Colombia, sin embargo, de estar ubicado en el 2013 en el puesto 139 logra en cinco años estar en el puesto 118 . 
Tabla 1.

Facilidades para hacer negocios

\begin{tabular}{|c|c|c|c|c|c|c|c|c|c|c|c|}
\hline $\begin{array}{r}\text { Ranki } \\
\text { ng } 2018 \\
\end{array}$ & Países & $\begin{array}{r}\text { Ranki } \\
\text { ng } 2017 \\
\end{array}$ & Países & $\begin{array}{r}\text { Ranki } \\
\text { ng } 2016 \\
\end{array}$ & Países & $\begin{array}{r}\text { Ranki } \\
\text { ng } 2015 \\
\end{array}$ & Países & $\begin{array}{r}\text { Ranki } \\
\text { ng } 2014 \\
\end{array}$ & Países & $\begin{array}{r}\text { Ranki } \\
\text { ng } 2013 \\
\end{array}$ & Países \\
\hline 55 & Chile & 53 & ia & 48 & Chile & 34 & ${ }_{\text {ia }}^{\text {Colomb }}$ & 34 & Chile & 37 & Chile \\
\hline 58 & Perú & 54 & Perú & 50 & $\begin{array}{l}\text { Perú } \\
\text { Colomb }\end{array}$ & 35 & Perú & 42 & $\begin{array}{l}\text { Perú } \\
\text { Colomb }\end{array}$ & 43 & $\begin{array}{l}\text { Perú } \\
\text { Colomb }\end{array}$ \\
\hline 59 & Colombia & 57 & Chile & 54 & ia & 41 & Chile & 43 & ia & 45 & ia \\
\hline 118 & Ecuador & 114 & Ecuador & 117 & Ecuador & 115 & Ecuador & 135 & Ecuador & 139 & Ecuador \\
\hline 152 & Bolivia & 149 & Bolivia & 157 & Bolivia & 157 & Bolivia & 162 & Bolivia & 155 & Bolivia \\
\hline 188 & Venezuela & 187 & $\begin{array}{l}\text { Venezu } \\
\text { ela }\end{array}$ & 186 & $\begin{array}{l}\text { Venezu } \\
\text { ela }\end{array}$ & 182 & $\begin{array}{l}\text { Venezu } \\
\text { ela }\end{array}$ & 181 & $\begin{array}{l}\text { Venezu } \\
\text { ela }\end{array}$ & 180 & $\begin{array}{l}\text { Venezu } \\
\text { ela }\end{array}$ \\
\hline
\end{tabular}

Fuente: Elaboración propia (basado en el Doing Business Report)

El marco conceptual del GEM es también una buena medida del efecto de las políticas públicas, considerando que se basa en el supuesto de que el crecimiento económico nacional es el resultado de las interdependencias entre las condiciones del marco emprendedor y los rasgos y capacidades personales de las personas para identificar y aprovechar las oportunidades (Lasio, Ordeñana, Caicedo, Samaniego, y Izquierdo, 2018).

El GEM desde 1999 entrega información uniforme acerca de la capacidad emprendedora y las principales características del emprendimiento, así como presenta un índice anual de actividad emprendedora para diversos países del mundo, en el caso del Ecuador se tomaron datos desde el año 2009 al 2017. El modelo consta de cuatro dimensiones fundamentales: un contexto social, económico y político; las condiciones nacionales generales; la estructura empresarial y de oportunidades; y los resultados generados por las nuevas empresas en el crecimiento económico, en el empleo y en la innovación (Carrillo y otros, 2014).

La tabla 2, recoge algunos indicadores importantes del país, se puede observar que las políticas gubernamentales en lo concerniente a regulación están por debajo de los países analizados y de las economías de eficiencia, así como el apoyo financiero, de igual manera los programas gubernamentales. Siendo puntos positivos, el indicador de infraestructura comercial y profesional y el de acceso a la infraestructura física. 
Tabla 2.

Evaluación del entorno

\begin{tabular}{|c|c|c|c|c|c|c|c|}
\hline Descripción & $\begin{array}{c}\text { Ecuad } \\
\text { or }\end{array}$ & Perú & $\begin{array}{l}\text { Chil } \\
\text { e }\end{array}$ & $\begin{array}{c}\text { Colomb } \\
\text { ia }\end{array}$ & $\begin{array}{c}\text { Regió } \\
\mathrm{n}\end{array}$ & $\begin{array}{l}\text { Economías de } \\
\text { eficiencia }\end{array}$ & $\begin{array}{l}\text { Glob } \\
\text { al }\end{array}$ \\
\hline & & 3.5 & 3.6 & & & & \\
\hline Apoyo financiero & 3.15 & 9 & 2 & 3.55 & 3.52 & 4.14 & 4.31 \\
\hline Políticas gubernamentales: Políticas & & 4.4 & 4.1 & & & & \\
\hline \multirow[t]{2}{*}{ generales } & 3.53 & 0 & 3 & 3.82 & 3.90 & 3.98 & 4.26 \\
\hline & & 3.4 & 4.5 & & & & \\
\hline \multirow[t]{2}{*}{ Políticas gubernamentales: Regulación } & 2.93 & 7 & 5 & 3.18 & 3.41 & 3.53 & 3.87 \\
\hline & & 4.5 & 5.1 & & & & \\
\hline Programas gubernamentales & 3.58 & 7 & 5 & 4.35 & 4.27 & 4.03 & 4.33 \\
\hline Educación y formación: Primaria y & & 3.6 & 2.5 & & & & \\
\hline secundaria & 3.60 & 5 & 1 & 3.61 & 2.73 & 3.01 & 3.20 \\
\hline Educación y formación: Educación & & 5.1 & 4.4 & & & & \\
\hline \multirow[t]{2}{*}{ superior } & 5.99 & 9 & 7 & 5.59 & 5.14 & 4.75 & 4.75 \\
\hline & & 3.5 & 3.4 & & & & \\
\hline \multirow[t]{2}{*}{ Transferencias de I+D } & 3.52 & 0 & 2 & 3.74 & 3.59 & 3.55 & 3.92 \\
\hline & & 4.3 & 4.1 & & & & \\
\hline \multirow[t]{2}{*}{ Infraestructura comercial y profesional } & 5.06 & 1 & 7 & 4.74 & 4.67 & 4.68 & 4.89 \\
\hline & & 4.2 & 4.1 & & & & \\
\hline \multirow[t]{2}{*}{ Apertura del mercado interior: Dinámica } & 4.59 & 2 & 4 & 4.51 & 4.41 & 5.12 & 5.08 \\
\hline & & 4.4 & 3.9 & & & & \\
\hline \multirow[t]{2}{*}{ Apertura del mercado interior: Apertura } & 3.93 & 5 & 0 & 4.17 & 3.93 & 3.98 & 4.20 \\
\hline & & 5.8 & 7.1 & & & & \\
\hline \multirow[t]{2}{*}{ Acceso a infraestructura física } & 6.99 & 4 & 0 & 6.19 & 6.19 & 6.33 & 6.45 \\
\hline & & 5.4 & 5.0 & & & & \\
\hline Normas sociales y culturales & 4.99 & 7 & 0 & 5.66 & 4.73 & 4.62 & 4.83 \\
\hline
\end{tabular}

Fuente: Elaboración propia (basado en el GEM Ecuador, 2017)

Las principales estrategias que se aplican en los países en donde se evidencian emprendimientos dinámicos se basan principalmente en la preparación educativa; aquí los individuos que deseen negocios que florezcan favorablemente con una mejor cultura emprendedora, crecimiento potencial y mantenimiento de réditos cuantiosos, deben poseer una mejor preparación educacional, especialmente universitaria y de áreas de negocios que fomenten y fortalezcan la actitud emprendedora que a posteriori dotará a dichos individuos de capacidades que les permita identificar nuevas oportunidades de negocios sin eximir los riesgos que presenten (Rivas, 2014).

Además, García, Martínez, y Onofre (2016) mencionan que para que exista dentro de los países emprendimiento dinámico, es necesario que impulsen las siguientes dimensiones:

$>$ Capital humano emprendedor, el cual se basa en el fortalecimiento de las condiciones sociales y el sistema educativo.

$>$ Condiciones de la demanda, que analiza las principales características del mercado, estructura empresarial y tecnología.

$>$ Financiamiento, la capacidad para acrecentar el capital y por ende mejorar los emprendimientos hasta llevarlos al nivel de dinámicos.

> Capital social, participación de los diferentes agentes sociales del sector empresarial, que permiten el establecimiento de redes para el acceso a recursos. 
Regulaciones y políticas, normas estatales que impulsen y motiven a emprender negocios dinámicos, que, a más de prevalecer en el tiempo, obtengan mejores beneficios económicos.

Sumándose a dichas dimensiones, es necesario que dentro de los países se fomenten políticas que permitan el desarrollo y crecimiento de las pequeñas empresas, tales como se detallan a continuación:

Políticas sectoriales de apoyo y fortalecimiento al emprendimiento dinámico
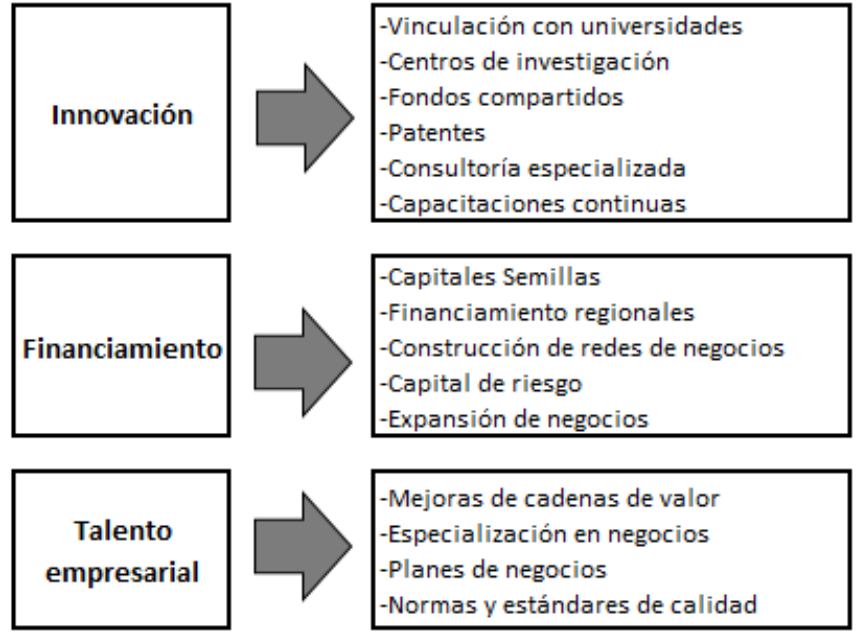

\begin{tabular}{|c|l|l|}
\hline $\begin{array}{c}\text { Políticas de } \\
\text { entorno }\end{array}$ & $\begin{array}{l}\text {-Marco legal de inversiones y capital } \\
\text {-Protección de las actividades } \\
\text {-Generación de ecosistemas favorables } \\
\text {-Creación de climas favorables de } \\
\text { inversión } \\
\text {-Apoyo de capital público } \\
\text {-Leyes incentivadoras de producción }\end{array}$ \\
\hline
\end{tabular}

Ilustración \#1 Políticas sectoriales de apoyo y fortalecimiento al emprendimiento dinámico. Las principales políticas deben fomentar especialmente la innovación, financiamiento, talento empresarial y las políticas del entorno, lo cual provocará que haya un mejor panorama para convertir emprendimientos convencionales en emprendimientos dinámicos. Adaptado de "Pyme de avanzada: Motor de desarrollo en América Latina", p.237, por

(Van Hoof y Gómez, 2015), Bogotá: Ediciones Uniandes.

\section{Conclusiones}

Este trabajo logra establecer que el emprendimiento sigue siendo desde los primeros aportes de autores clásicos, un elemento clave en el crecimiento económico de los países, sin embargo, los estudios recientes lo ubican en torno a la necesidad de contar con emprendimientos de gran crecimiento o de gran escalabilidad por su fuerte impacto en la creación de empleo.

En la actualidad, el emprendimiento viene siendo estudiado de manera muy profunda en los países, considerando el ecosistema emprendedor en investigaciones cada vez más complejas con el objetivo de apoyarlo por su relación con la creación de empleo. Así pues, en la última década del siglo XX en cuanto a la actividad emprendedora y su relación la creación de nuevas empresas, hay estudios que favorecen la presencia de los gobiernos con marcos regulatorios 
favorables. Es así que muchos gobiernos son desde el año 1999 adherentes al proyecto GEM con el objeto de poder medir la actividad emprendedora en sus países.

Por otra parte, parece evidente la existencia de una relación positiva entre la actividad emprendedora y el crecimiento económico, a tal punto, que se puede observar como las políticas públicas tomadas desde el 2010 han generado un entorno favorable para mejorar el posicionamiento del Ecuador en la forma de hacer negocios.

En este contexto, y dada la grave y larga crisis económica, con agudas consecuencias sociales, por la que atraviesa nuestro país desde el año 2008, la recién aprobada Ley 14/2013, de 27 de septiembre, de apoyo a los emprendedores y su internacionalización, pretende invertir esta situación, contribuyendo al cambio de mentalidad necesario para que el entorno sea más favorable al emprendedor y que la sociedad valore más.

\section{Bibliografía}

Arteaga, M., \& Lasio, V. (2009). Empresas dinámicas en Ecuador: factores de éxito y competencias de sus fundadores. Academia, Revista Latinoamericana de Administración, $1-19$. Obtenido de https://www.researchgate.net/publication/41805459_Empresas_dinamicas_en_Ecuador_f actores_de_exito_y_competencias_de_sus_fundadores

Asamblea Nacional del Ecuador. (2012). Ley Orgánica de Regulación y Control del Poder de Mercado.

Audretsch, D. B., \& Fritsch, M. (2002). Growth Regimes over Time and Space. Regional Studies, 36(2), 113-124. http://doi.org/10.1080/00343400220121909

Campuzano, J., Espinoza, E., \& Cedillo, L. (2017). La actividad emprendedora en la provincia de El Oro, una revisión desde la universidad. Universidad y Sociedad, 73-81. Obtenido de https://rus.ucf.edu.cu/index.php/rus/article/view/610/716

Cantón, L., García, A., \& Iuit, M. (2014). El Enfoque de Competencias para formar Emprendedores Evaluación del modelo de la Universidad Autónoma de Yucatán, México. Tec Empresarial, 29-40. Obtenido de https://dialnet.unirioja.es/servlet/articulo?codigo $=4808515$

Carrillo, L. L., Priede, T., \& López-Cózar, C. (2014). El emprendimiento como motor del crecimiento económico. Boletín Económico de ICE, Información Comercial Española.

Coad, A., Daunfeldt, S.-O., Hölzl, W., Johansson, D., \& Nightingale, P. (2014). High-growth firms : introduction to the special section. Industrial and Corporate Change, 23(1), 91112. http://doi.org/10.1093/icc/dtt052

Chong, E. (2016). Diseño y aplicación de un modelo de validación del nivel de madurez y potencial de crecimiento de los emprendimientos en la etapa de gestación. Quality Leadership University, 80-105. Obtenido de http://qlu.ac.pa/clee/wpcontent/uploads/2016/04/Memorias-del-CLEE-2016.pdf\#page=80

Dini, M., Rovira, S., \& Stumpo, G. (2014). Una promesa y un suspirar. Políticas de innovación para pymesen América Latina. Santiago de Chile: Naciones Unidas. Obtenido de https://repositorio.cepal.org/bitstream/handle/11362/37352/S1420481_es.pdf?sequence=1 \&isAllowed $=\mathrm{y}$ 
Fischer, E., \& Reuber, R. (2003). Support for Rapid Growth Firms: A Comparison of the Views of Founders, Government Policy Makers and Private Sector Resource Providers. Journal of Small Business Management, 346-365. Obtenido de http://citeseerx.ist.psu.edu/viewdoc/download?doi=10.1.1.201.8926\&rep=rep1\&type=pdf

Fritsch, M., \& Mueller, P. (2004). Effects of New Business Formation on Regional Development over Time. Regional Studies, 38(8), 961-975. http://doi.org/10.1080/0034340042000280965

Fuentelsaz, L., \& González, C. (2015). El fracaso emprendedor a través de las instituciones y la calidad del emprendimiento. Universia Business Review, 64-81. Obtenido de https://ubr.universia.net/article/view/1527

Garcés, F. (Abril de 2015). Repositorio de la Universidad Andina del Ecuador. Obtenido de Definición de los lineamientos generales de una política pública para el fomento del emprendimiento en el Ecuador: http://repositorionew.uasb.edu.ec/bitstream/10644/4461/1/T1613-MGDE-GarcesDefinicion.pdf

García, B., Martínez, M. D., \& Onofre, J. (2016). Condiciones sistémicas para el emprendimiento dinámico en América Latina. Boletín Científico de las Ciencias Económico Administrativas del ICEA. Obtenido de https://repository.uaeh.edu.mx/revistas/index.php/icea/article/view/240/3862

Hidalgo, G., Kamiya, M., \& Reyes, M. (2014). Emprendimientos dinámicos en América Latina. Avances en prácticas y políticas. Corporación Andina de Fomento. Obtenido de http://scioteca.caf.com/bitstream/handle/123456789/371/emprendimientos_dinamicos_a merica_latina.pdf?sequence $=1 \&$ isAllowed $=y$

Kantis, H., \& Díaz, S. (2008). Nuevas Empresas Dinámicas y de Alto Potencial de Crecimiento. En H. Kantis, \& S. Díaz, "Innovación y Emprendimiento en Chile: una radiografía de los emprendedores dinámicos y de sus prácticas empresariales” (pág. 18). Santiago: Endeavor Chile. Obtenido de http://www.celadel.org/textos/santiago/innovacion.pdf

Kantis, H., Angelelli, P., \& Moori, V. (2004). Desarrollo Emprendedor, América Latina y la experiencia internacional. Washington: Banco Interamericano de Desarrollo. Obtenido de http://www.academia.edu/35113213/2004_BID_Desarrollo_Emprendedor_AL.pdf

Kantis, H., Federico, J., \& Ibara, S. (2014). Índice de Condiciones Sistémicas para el Emprendimiento Dinámico Una herramienta para a acción en América Latina. E-Book. Obtenido de http://www.endeavor.cl/wp-content/uploads/2014/07/\%C3\%8dndice-decondiciones-sistemicas-para-el-emprendimiento-dinamico.pdf

Kantis, H., Gonzalo, M., \& Álvarez, P. (2014). ¿Emprendedores “ambiciosos” en Argentina, Chile y Brasil? el Papel del Aprendizaje y del Ecosistema en la Creación de Nuevas Empresas Dinámicas. Pymes, Innovación y Desarrollo, 33-60. Obtenido de https://dialnet.unirioja.es/servlet/articulo?codigo $=6353893$

Kantis, H., Gonzalo, M., Federico, J., \& Ibarra, S. (2014). En H. Kantis, M. Gonzalo, J. Federico, \& S. Ibarra, ¿Emprendimientos dinámicos en América del Su?: La claves es el (eco)sistema (pág. 24). Montevideo: red mercosur de investigaciones económicas. obtenido de https://www.researchgate.net/publication/303207113_emprendimientos_dinamicos_en_a merica_del_sur_la_clave_es_el_ecosistema

Lasio, V., Ordeñana, X., Caicedo, G., Samaniego, A., \& Izquierdo, E. (2018). Global Entrepeneurship Monitor Ecuador 2017. (E.- ESPOL, Ed.). Guayaquil.

Minniti, M. (2012). El emprendimiento y el crecimiento económico de las naciones. Economía

Esta obra se comparte bajo la licencia Creative Common Atribución-No Comercial 4.0 International (CC BY-NC 4.0) Revista de la Universidad Internacional del Ecuador. URL: https://www.uide.edu.ec/ 
Industrial, 1(383), 23-30. http://doi.org/10.15174/au.2014.701

Messina, M., \& Hochsztain, E. (2015). Factores de éxito de un emprendimiento: Un estudio exploratorio con base en técnicas de Data Mining. TEC Empresarial, 31-40. Obtenido de http://revistas.tec.ac.cr/index.php/tec_empresarial/article/view/2206

Montoya, D. (2015). Startups: tendencias en América Latina y su potencialidad para el crecimiento empresarial. Contexto, 7-20. $\quad$ Obtenido de http://revistas.ugca.edu.co/index.php/contexto/article/view/416/733

Morales, M. (2009). El clima de emprendimiento, un determinante clave en la intención emprendedora de los estudiantes de Escuelas de Negocio. Asamblea Anual Cladea 2009, 1-16. Obtenido de http://www.ascolfa.edu.co/web/archivos/conferencia2009/memorias/MemoriasCladea200 9/upac01_submission_451.pdf

Oyarvide, H., Nazareno, I., Roldán, A., \& Ferrales, Y. (2016). Emprendimiento como factor del desarrollo turístico rural sostenible. Retos de la Dirección, 10(1), 71-93. Obtenido de http://scielo.sld.cu/pdf/rdir/v10n1/rdir06116.pdf

Padilla, M., Lascano, L., \& Ramiro, W. (2018). La dinámica empresarial y el emprendimiento, factores determinantes para el desarrollo del ciclo de vida de las pymes. Revista Publicando, 308-325. Obtenido

de https://www.rmlconsultores.com/revista/index.php/crv/article/view/1376/pdf_1007

Palmero, C., Escolar, C., Luis, I., de la Torre, T., Gañán, Á., Baños, V., . . . Jiménez, A. (2014). Impacto de la educación en el emprendimiento. Making-of de un análisis por grupos de discusión. International Journal of Developmental and Educational Psychology, 507-518. Obtenido

de http://infad.eu/RevistaINFAD/OJS/index.php/IJODAEP/article/view/821/761

Rivas, G. (2014). El fomento al emprendimiento dinámico en los países del Cono Surde América Latina. Análisis de experiencias y lecciones de políticas. Banco Interamericano de Desarrollo. Obtenido https://publications.iadb.org/bitstream/handle/11319/6646/DP\%20CTI\%20El\%20foment o\%20al\%20emprendimiento\%20din\%C3\%A1mico\%20en\%20pa\%C3\%ADses\%20del\% 20Cono\%20Sur\%20de\%20Am\%C3\%A9rica\%20Latina.pdf?sequence=1\&isAllowed=y

Sánchez, Y., García, F., \& Mendoza, J. (2015). La capacidad de innovación y su relación con el emprendimiento en las regiones de México. Estudios Gerenciales, 243-252. Obtenido de https://www.sciencedirect.com/science/article/pii/S0123592315000315

Silveira, Y., Cabeza, D., \& Fernández, V. (2016). Emprendimiento: perspectiva cubana en la creación de empresas familiares. European Research on Management and Business Economics, 70-77. Obtenido de https://www.sciencedirect.com/science/article/pii/S1135252315000490

Van Hoof, B., \& Gómez, H. (2015). Pyme de avanzada: Motor de desarrollo en América Latina. Bogotá: Ediciones Uniandes. Obtenido de https://books.google.com.ec/books?hl=es\&lr=\&id=KdhdDwAAQBAJ\&oi=fnd\&pg=PA2 $23 \& d q=e m p r e n d i m i e n t o+d i n \% C 3 \% A 1$ mico\&ots=uztGZkQmmv\&sig=TxbHkTFbF8BM SvplnRFvXaf7SS0\&redir_esc $=\mathrm{y} \# \mathrm{v}=$ onepage $\& \mathrm{q} \& \mathrm{f}=$ false 